\title{
Anabases
}

ANABASES Traditions et réceptions de l'Antiquité

$17 \mid 2013$

Varia

Quod aliquando fuit, potest instaurari : parler latin au $\mathrm{XVI}^{\mathrm{e}}$ siècle, une restitution en trompe-l'œil?

\section{Martine Furno}

\section{OpenEdition}

Journals

Édition électronique

URL : http://journals.openedition.org/anabases/4146

DOI : 10.4000/anabases.4146

ISSN : 2256-9421

\section{Éditeur}

E.R.A.S.M.E.

\section{Édition imprimée}

Date de publication : 1 mars 2013

Pagination : 105-118

ISSN : 1774-4296

\section{Référence électronique}

Martine Furno, «Quod aliquando fuit, potest instaurari : parler latin au xvi ${ }^{e}$ siècle, une restitution en trompe-l'œil ? », Anabases [En ligne], 17 | 2013, mis en ligne le 01 avril 2016, consulté le 21 octobre 2019. URL : http://journals.openedition.org/anabases/4146 ; DOI : 10.4000/anabases.4146 
Anabases 17 (2013), p. 105-118.

\section{Quod aliquando fuit, potest instaurari: parler latin au XVI ${ }^{\mathrm{e}}$ siècle, une restitution en trompe-l'œil ?}

Martine Furno

L'une des Restitutions de l'Antiquité à LA Renaissance passe par la maîtrise de la langue latine, maitrise qui ne saurait être complète sans ce que nous appelons aujourd'hui les trois compétences nécessaires à la pratique d'une langue vivante, autrement dit le latin écrit, lu et parlé. Sur ces trois capacités, celle qui a fait le plus souvent défaut ou débat est celle de l'oral : en effet plus aucun locuteur natif n'est susceptible à cette époque de transmettre la langue, et les doctes les plus brillants l'ont forcément apprise comme une langue de culture dont il n'ont pas d'usage quotidien. Le latin n'est plus, au XVI e siècle, un sermo maternus, à tous les sens du terme, c'est-à-dire ni une langue véhiculaire qui s'apprend par la mère, ni ce que nous appelons au sens moderne une langue maternelle : pour tous il s'agit d'une langue seconde, quel que soit le degré de maittrise auquel certains parviennent, et quelles que soient les tentatives de restituer cette langue comme oral véhiculaire de la République des Lettres.

Nous verrons donc en un premier temps que ceux qui parlent latin au XVI ${ }^{\mathrm{e}}$ siècle ne sont que rarement des doctes en situation de communication, et bien plus souvent des maitres et des élèves en situation pédagogique. En réduisant l'enquête à ce champ qui est le seul à être sérieusement documenté, nous tenterons un état des lieux de la prononciation et de la pratique de la langue, à travers des textes d'Érasme, de Charles Estienne et de Mathurin Cordier, dont les Colloquia scholastica peignent le petit thêâtre d'une institution scolaire qui nous a transmis bien des habitudes. 


\section{Parler latin pour apprendre le latin}

Qui parle latin au XVI siècle, et quand ? Si, dans la continuité d'une pratique savante ou cléricale au Moyen Âge, la fin du XV siècle a parlé le latin comme langue de communication entre membres de la république des Lettres, cette restitution s'est heurtée au fait que la pratique orale, que les doctes en conviennent ou pas, était nécessairement non quotidienne, discontinue, et difficile ${ }^{1}$.

Les doctes et les pédagogues du XVI ${ }^{\mathrm{e}}$ siècle ne peuvent guère faire un autre constat. Hormis des cas particuliers et extrêmement ponctuels de communication entre savants, aucune occasion naturelle de parler latin n'est offerte au quotidien dans la vie familiale ou sociale. La seule situation récurrente de pratique orale est une situation pédagogique délibérée, pour restituer les conditions d'apprentissage de l'Antiquité et obtenir avec les mêmes commodités un même résultat de maîtrise orale. Des enseignants, aussi bien que des parents avisés, tentent donc de créer des situations d'apprentissage par immersion, persuadés qu'on ne peut apprendre facilement le latin qu'en le parlant, comme les anciens Romains :

"Car c'est en parlant bien que nous apprenons en même temps à bien écrire. Vice versa,

c'est en écrivant soigneusement que nous nous habituons aussi à parler correctement ${ }^{2}$."

constatent avec conviction deux des jeunes personnages des Colloquia de Cordier.

C'est de la même idée que procède le "roman pédagogique " que nous raconte Montaigne au chapitre XXV du livre 1 des Essais, lorsqu'il nous détaille " l'institution exquise " que son père choisit pour lui faire apprendre le latin « sans art, sans livre, sans grammaire ou précepte, sans fouet; et sans larmes ». M. de Montaigne donc, constatant qu'on " achète trop cher » l'apprentissage du grec et du latin pour les enfants, décida de confier son fils dès le berceau à un précepteur allemand et latinophone, qui lui parlerait latin constamment, ainsi que toutes les personnes qui seraient en contact avec lui, recréant ainsi les conditions d'immersion du sermo maternus. Les effets de cette méthode

1 Le débat même sur la "question de la langue ", qui agite les milieux italiens lettrés entre 1435 et la fin du siècle, et porte sur le fait de savoir quel latin parlaient exactement les Romains et notamment les "Romains de la rue ", est en fait implicitement né d'une constatation pédagogique désabusée. Partant de la réalité $\mathrm{du} \mathrm{Xv}^{\mathrm{e}}$ siècle où il est difficile aux enfants d'apprendre le latin, certains en conséquence ont jugé quasi impossible que des illitterati comme les gens de la plèbe, même à Rome, aient pu le parler de manière grammaticalement correcte. D'où l'idée pour certains, comme Leon Battista Alberti notamment, que la plèbe romaine ait pu parler un latin en quelque sorte dégrammatisé qui serait la source directe du vernaculaire italien. Sur ce débat complexe et foisonnant, voir M. TAVoni, Latino, grammatica, volgare, Storia di una questione umanistica, Padova, Antenore, 1985.

2 Mathurin Cordier, Colloquiorum scholasticorum libri quatuor, Genève, Henri Estienne, 1564, livre 4, coll. 19, p. 147 : Nam bene loquendo, bene etiam scribere condiscimus. Contra, diligenter scribendo consuescimus etiam recte loqui. 
selon Montaigne furent merveilleux, puisqu'une fois au collège de Guyenne de grands doctes comme Nicolas Grouchi, Georges Buchanan et Marc-Antoire Muret « craignaient à [l] accoster $^{3}$ ". Nous verrons les restrictions qu'il faut peut-être apporter à cette idyllique restitution, qui prend dans le souvenir de l'adulte savant l'allure d'une " merveille » : mais pour exceptionnelle qu'elle soit dans sa réalisation, cette méthode procède de la même idée que celle qui s'exprimera à la fin du siècle dans les Colloquia de Cordier.

Un autre témoignage du même type, que nous possédons sous deux formes même si les deux textes ne me semblent pas avoir été mis en parallèle, nous permet quelques précisions sur ces situations particulières. En effet, l'autre seul texte que je connaisse qui nous détaille une maison où la conversation latine est chose quotidienne est la préface qu'Henri Estienne place, sous la forme d'une lettre à son fils Paul, au début de son édition d'Aulu-Gelle de 1585. Il y décrit à son fils, comme un modèle d'érudition et d' "incitation domestique à l'étude des lettres ", la maison de son père Robert Estienne, où, pour se comprendre entre eux, les correcteurs et hommes savants de différentes langues vernaculaires qui logent dans l'atelier utilisent le latin comme truchement, et entraînent toute la maisonnée à maintenir cette langue comme langue de communication, également avec les femmes et la domesticité. Le texte d'Henri Estienne est plus nuancé que celui de Montaigne, car il précise bien que les domestiques se limitent à une langue nécessaire à leur fonction, et que l'on mêle parfois du français au latin : mais les deux fils de Robert, Henri et son frère Robert II, "n'os[aient] pas [se] servir d'autre langue que le latin avec [leur] père " et leur sœur Catherine, même si elle malmène un peu la grammaire, peut s'exprimer dans la langue de Cicéron sans que " rien d'autre que l'usage ne lui [ait] ouvert la voie. En effet elle a appris le latin de la même façon qu'on a l'habitude d'apprendre le français chez les Français, l'italien chez les Italiens, et les autres langues chez les autres peuples ${ }^{4}$ ».

On voit ici comment une situation de communication entre doctes se lie, dans le souvenir de l'adulte savant, à une situation pédagogique idéale d'apprentissage par immersion. C'est sans doute la même maison Estienne, qui, sans être nommée, est représentée dans un des colloques de Cordier : ami intime de Robert I Estienne, Cordier fréquente son atelier et sa maison depuis la période où le jeune Henri " commence à balbutier dans cette langue » latine, et il s'est plu dans le colloque 44 du livre 2 à donner sa propre version, elle aussi idéalisée pédagogiquement, de cette famille savante. Dans ce texte en effet, Eusebius s'étonne que le petit frère, tout juste âgé de cinq ans, de son camarade Montanus, sache déjà parler latin. Montanus lui décrit alors l'organisation familiale : un pédagogue savant ne parle que latin aux enfants, selon la consigne d'un

3 Montaigne, Essais, Livre 1, chapitre XXV, De l'Institution des enfants, Paris, La Pochothèque, 2001, p. 267-270 (texte de l'édition de 1595).

4 H. Estienne, Lettre à P. Estienne, préface de l'édition d'Aulu-Gelle par H. Estienne, Paris, 1585, éditée dans La France des humanistes, Henri II Estienne éditeur et écrivain, sous la dir. de J. CÉARD, Turnhout, Brepols, 2003, p. 533-534. 
père " qui se soucie de [les] éduquer avec tant de soin ", père auquel les enfants n'osent s'adresser qu'en latin. Le français est réservé aux conversations avec leur mère et avec les servantes, tandis que les serviteurs leur parlent aussi latin. Cordier a repris ici l'organisation de la famille Etienne, en sublimant lui aussi la situation particulière d'érudition de cette famille en un parangon éducatif où le but de la restitution orale du latin est bien plus l'apprentissage de la langue que la communication savante ${ }^{5}$.

La situation des deux familles Montaigne et Estienne est exceptionnelle : s'il est intéressant de noter que cette tentative pédagogique apparaît dans deux milieux sociaux différents avec la même idée d'économiser en quelque sorte le temps d'étude des enfants, il ne faudrait pas pour autant en généraliser l'existence. La plupart des garçons apprennent le latin au collège, collectivement, et quelques textes nous rendent compte de ces efforts pour un oral correct et efficace. La tâche n'est pas mince : les difficultés concernent d'abord la prononciation, comme nous le verrons en parcourant le De recta latini graecique sermonis pronunciatione d'Érasme, paru en 1528 à Bâle chez Froben, et son prolongement, le De recta latini sermonis pronunciatione scripturaque de Charles Estienne, paru chez François Estienne en $1541^{6}$. Grammaire et stylistique nous occuperont ensuite, à travers l'analyse de quelques textes pédagogiques de Mathurin Cordier.

\section{La prononciation du latin ou les difficultés d'une restitution crédible}

Le De recta latini graecique sermonis pronunciatione d'Érasme est un traité savant, rédigé en 1528 à destination des maitres pour qu'ils apprennent eux-mêmes à parler correctement le latin, avant de le transmettre à leurs élèves. Le traité, qui s'ouvre sur une sorte de sociologie de l'enseignant au XVI ${ }^{\mathrm{e}}$ siècle, dresse un assez triste état de la performance orale, rendue difficile pour Érasme par l'influence des vernaculaires et des accents nationaux, et par le fait que l'on apprend cet oral par un écrit qui ne transcrit pas la prononciation : sans alphabet phonétique et sans locuteur modèle, chacun oralise comme il peut ${ }^{7} .$. Quelques

5 Un des parallèles les plus frappants entre les deux textes est la remarque des enfants qui " n'osent pas " parler à leur père autrement qu'en latin : Patrem non audemus nisi Latine alloqui, dit le personnage de Cordier, et Henri Estienne rappellera Ego fraterque meus Robertus Stephanus non alia quam Latina apud patrem... uti Lingua auderemus. Voir le texte de M. Cordier en annexe.

6 A.A. Renouard (Annales de l'imprimerie des Estienne, Paris, 1843, p. 90) identifie une seule édition de cet ouvrage, imprimé sans nom d'auteur sur la page de titre, par François et Robert Estienne en 1538. La préface cependant, signée Charles Estienne, désigne son auteur. Une édition plus courante que celle de 1538 semble être celle de 1541 par François Estienne, accessible notamment en ligne sur Gallica, http://gallica.bnf.fr/ ark :/12148/bpt6k5455264h.

7 Érasme, De recta latini graecique sermonis pronunciatione, édition utilisée : Paris, Robert Estienne, 1547, p. 61 : Nunc enim, tota fere pronuntiatio deprauata est, tum apud Graecos, 
années plus tard, en 1538, Charles Estienne reprend les principaux préceptes d'Érasme dans un manuel beaucoup plus court et directement pratique, mais où les préoccupations sont identiques. Les défauts que l'un et l'autre soulignent sont essentiellement ceux de la perte d'une accentuation correcte, faute de perception de la différence entre longues et brèves, ainsi que des défauts de prononciation des phonèmes, provoqués cette fois par les habitudes orales des vernaculaires.

Le premier de ces points, c'est-à-dire l'accentuation, est détaillé précisément chez Érasme. Celui-ci y fait la différence entre la distinction des voyelles longues et brèves en elles-mêmes, et l'accentuation du mot, la seconde étape étant partiellement générée par la première, mais les deux phénomènes restants distincts. Érasme moque les intelligences épaisses

" qui ne distinguent pas l'accent de la quantité, alors que l'organisation en est bien différente. C'est une chose en effet qu'un accent aigu, autre chose que tenir longtemps un son, de même que c'est une chose que d'augmenter, une autre que d'allonger, quoique rien n'empêche que la même syllabe ait à la fois un accent aigu et un son long comme dans les passés Vidi et $\operatorname{Legi}^{8}{ }$.

Mais en 1541 dans le traité pédagogique d'Estienne, qui vise à l'efficacité, ces complexités sont sensiblement réduites. Estienne renonce à détailler les règles de longueur qui engendrent les règles d'accentuation, et préfere s'en tenir à la mémorisation directe de l'accent lui-même, par la mémorisation des différentes combinaisons qui le génèrent. Cette méthode est l'aveu implicite d'un renoncement, celui de faire entendre les longueurs, probablement parce que ses lecteurs ne les percevront pas : on peut se demander si, ainsi limité à un accent tonique uniquement marqué graphiquement par un aigu, le latin est véritablement restitué, ou simplement transposé à la mesure des capacités modernes d'oralisation? .

tum apud Latinos. - [...] Vnde nam accidit? - Partim ex corruptela unlgati sermonis, partim hinc quod soni uocum scribi non queunt. ("Désormais en effet presque toute la prononciation est dépravée, en Grec comme en Latin. - D’où cela vient-il ? - En partie à cause de la corruption du vernaculaire, en partie du fait que les sons ne peuvent se transcrire. ")

Érasme, De recta... pronunciatione, p. 75 : Sunt quidam adeo crassi, ut non distinguant accentum a quantitate, quum sit longe diuersa ratio. Aliud est enim acutum, aliud diu tinnire: sicut aliud intendi, aliud extendi, quanquam nibil uetat eandem syllabam et acutum habere tonum, et productum tempus, uelut in Legi et Vidi praeteritis.

9 La question de l'accent occupe les $\mathrm{f}^{\circ} 7 \mathrm{v}^{\circ}$ à $17 \mathrm{v}^{\circ}$, sur les 23 que comporte le manuel proprement dit. Estienne y passe en revue la façon d'accentuer les mots par catégorie grammaticale (noms propres, communs, pronoms, verbes, participes, conjonctions, prépositions et interjections). Le principe consiste à poser une règle, des exemples, et si nécessaire à signaler quelques exceptions : par exemple et au hasard, $\mathrm{f}^{\circ} 8 \mathrm{v}^{\circ}:$ AL. Al finita si sint appellatiua, et consonans antecedat, acuuntur in penultima. Vectígal, Tribuinal. Excipitur ánimal. Barbara autem propria, accentum habent in antepenultima. A'nnibal. A'sdrubal. ( AL. Les noms communs terminés en AL précédé d'une consonne sont accentués sur la 
Les deux textes d'Érasme et Estienne convergent également sur la question des vices phonétiques. L'un et l'autre soulignent combien les accents nationaux pèsent sur la prononciation des voyelles ou des consonnes, jusqu'à rendre l'oral inintelligible pour du latin, ou totalement ridicule, comme le raille Érasme. Il raconte en effet une anecdote où l'on voit l'empereur Maximilien salué en latin par quelques orateurs, dont un Français. Celui-ci, bien qu'il lût un discours plutôt de bonne facture qui d'ailleurs, glisse Érasme, lui avait sans doute été écrit par un Italien, le prononça tellement à la française que quelques Italiens présents crurent qu'il parlait français, non latin. Par raillerie, les orateurs suivants grossissent alors eux-mêmes les traits de leur prononciation nationale, et Érasme s'amuse à transcrire des maghestas, fidere fos, et lipenter à l'allemande notamment ${ }^{10}$. Or Charles Estienne constate toujours les mêmes traits une quinzaine d'années plus tard, lorsqu'il reprend, avec moins de causticité certes, les Allemands qui disent Ponus pour Bonus, Tebeo pour Debeo, ou les Français qui font disparaître les aspirées et confondent habeo et $a b e o^{11}$. À lire ces deux textes, on peut se demander quel diable de latin parlait Montaigne, appris tout pur de la bouche de son précepteur allemand et de ses domestiques gascons...

pénultième : Vectígal, Tribúnal. Exception : ánimal. Mais les noms propres barbares ont l'accent sur l'antépénultième : \{A'nnibal. A'sdrubal. »)

10 Érasme, De recta... pronunciatione, p. 138-139: Non admodum diu est, quod me forte praesente, Maximilianus Caesar ab aliquot oratoribus salutaretur... quorum unus erat Gallus... Is orationem ab Italo quopiam, ut arbitror, compositam, nec male Latinam, adeo Gallice pronuntiauit, ut Italis aliquot eruditis qui tum aderant Gallice non Latine dicere crederetur. [...] Quaerebatur qui ex more responderet, idque ex tempore... Protusus est ad hoc negotii Doctor quidam aulicus. [...] Is hunc in modum orsus est, Caesarea maghestas pene caudet fidere fos, et horationem festram lipenter audifit ; Aliaque tanto spiritu, tamque germanice, ut nemo uulgari lingua dicens possit magis Germanice. Hunc maior excepit risus. Sequutus est orator Daniae, quamquam is qui dicebat, uidebatur Scotus, mire referens eius gentis pronuntiationem. Huic oppositus est Zelandus quidam : deierasses neutrum loqui latine. ("Il y a quelque temps, alors que j'étais présent par hasard, l'empereur Maximilien était salué par quelques orateurs, [...] dont l'un était français. Celui-ci lut un discours composé, à mon avis, par un Italien, plutôt en bon latin, mais il le prononça tellement à la française que quelques Italiens savants qui étaient présents crurent qu'il le disait en français, non en latin. [...] on se demandait qui répondrait de la même façon, et aussitôt... Un conseiller aulique se leva pour cela. Il commença de cette manière, Caesarea maghestas pene caudet fidere fos, et horationem festram lipenter audifit, et autres, avec tant d'esprit et de manière tellement germanique que personne n'aurait pu le faire plus germaniquement en parlant le vernaculaire. Il s'ensuivit un grand rire. Suivit un orateur Danois, qui cependant semblait Écossais, rapportant de façon étonnante la prononciation de ce peuple ; un Hollandais lui répondit : tu aurais dit que ni l'un ni l'autre ne parlaient latin.")

11 Charles Estienne, De recta latini sermonis pronuntiatione et scriptura, Paris, François Estienne, 1541, $\mathrm{f}^{\circ} 19 \mathrm{r}^{\circ}$ : Vitium item committitur, si quando p pro b efferatur quae prolatio Germanis est peculiaris, ponus pro bonus: $\mathrm{f}^{\circ} 19 \mathrm{v}^{\circ}$ : D literam germanus vulgus minus recte profert, dum Tebeo pro Debeo... sonat; $\mathrm{f}^{\circ} 20 \mathrm{v}^{\circ}$ : Omittunt praecipue nostri Galli in scribendo et loquendo, dum nibil discriminis constituere videntur inter habeo et abeo. 


\section{La pratique de la langue : les ambiguïtés d'une réussite}

Il faut donc souvent s'exercer et se corriger lorsqu'on prononce le latin, et l'apprentissage par l'oral suppose de progresser par erreurs et corrections pour la pratique syntaxique et stylistique. Parmi les divers témoignages que nous pouvons trouver sur ce point dans le courant $\mathrm{du} \mathrm{XVI} \mathrm{e}^{\mathrm{e}}$ siècle, les plus édifiants sont sans doute ceux de Mathurin Cordier, personnage attachant qui a voué sa vie à l'enseignement du latin dans les petites classes, et nous a laissé plusieurs manuels éclairants sur ce qui se passait dans les collèges.

Mathurin Cordier, né en France en 1474 ou 1479, enseigne d'abord la rhétorique dans les collèges parisiens, jusqu'au jour où, désespéré du piètre niveau de ses élèves en latin, il décide de se charger lui-même des petites classes, ce qui lui vaudra la reconnaissance de Calvin à qui il apprend le latin au collège de La Marche. Ami de Robert Estienne qui lui ouvre la voie de l'évangélisme, il s'exilera comme lui à Lausanne et Genève, où il finira sa vie en ayant repris une classe, à plus de quatre-vingts ans, sans doute pour des raisons financières mais aussi par amour de la pédagogie. Cordier n'est pas séduit dès le début de sa carrière par l'imprimerie : c'est peut-être sous l'influence de Robert Estienne qu'il accepte d'imprimer en 1530 le De corrupti sermonis latini emendatione, ouvrage qui est un antibarbarus, destiné à corriger les erreurs de langue commises par les enfants, et que Cordier a relevées après les avoir entendues dans les classes ${ }^{12}$.

L'ouvrage de Cordier est pour nous un document de choix sur l'oral pédagogique : en effet il nous donne simultanément les tournures françaises utilisées dans la conversation scolaire, leur traduction fautive et la traduction préconisée ${ }^{13}$, ce qui nous permet d'identifier les difficultés des élèves et les remèdes proposés. Cordier travaille sur la varietas et la copia du vocabulaire, en donnant des équivalents systématiques à la plupart des expressions concernées ${ }^{14}$, mais aussi sur la correction syntaxique et stylisti-

12 Sur Mathurin Cordier et l'imprimé, voir M. Furno, «Du cours volé au testament pédagogique : Mathurin Cordier et le choix de l'imprimé ", à paraître dans les actes du Colloque Repenser l'imprimé, Toronto, octobre 2010.

13 Construit ainsi, le livre n'était pas une bonne idée pédagogique : en 1541, Cordier en donne une refonte, toujours chez Robert Estienne, sous le titre Commentarius puerorum de quotidiano sermone, dans laquelle il a notamment retiré le latin fautif. En effet, certains professeurs refusaient d'utiliser le livre en classe, car les élèves, plutôt que de s'en tenir aux exemples de bon latin, se plaisaient à utiliser les tournures macaroniques...

14 Pour un exemple parmi tant d'autres de cette méthode, voir Mathurin Cordier, De corrupti sermonis emendatione, chapitre 2, $\$ 160$ (éd. Robert Estienne, Paris, 1536, in- $4^{\circ}$, p. 27) : pour l'expression française «Iauroye beau effeuilleter tout le liure pour trouver ung mot ", Cordier propose le latin Longum est totum codicem euoluere, vt unicum verbum inueniam, puis explicite la synonymie par Varietas : Voluerre, Euoluere; ensuite il propose un équivalent dans le même registre de sens pris à Térence, et traduit : Terentius : Longum est nos illum expectare dum exeat, Nous aurions bel attendre tant qu'il sorte, [cf. Térence Andrienne, 977 : atque adeo longumst illum me exspectare dum exeat] avec une variante possible de mise en situation : Longum est me expectare te, dum redeas, Iauroye beau uous 
que. Sur ce point, les défauts principaux et récurrents sont générés par le vernaculaire, puisque beaucoup des tournures qui doivent être corrigées sont en fait des calques du vernaculaire qui aboutissent à un latin bariolé... Par exemple, pour l'expression des compléments de temps marquant la durée, Cordier a relevé les tournures suivantes :

Quot bene est te esse intus? Quantum est quot tu manes intus? Quanti est te esse intus? Combien y a il que tu demeures cyens? Sunt duo anni.

Ce latin est évidemment marqué par diverses erreurs dues à la transposition directe du français, au mépris du sens (Quot bene pour combien...) ou des règles de grammaire (le nominatif Sunt duo anni dans la réponse). Cordier propose alors les corrections suivantes :

Quam pridem hoc habitas? Duos annos, Id est iam duo sunt anni, Il y a deux ans,

avec l'emploi correct d'un accusatif de durée ou du marqueur temporel iam. Il ajoute à cette base diverses autres tournures pour enrichir l'expression ou corriger d'autres erreurs du même champ sémantique :

post duos annos, depuis deux ans, quod barbare dicitur, de post duos annos;

Ante decem annos, Il y a dix ans passez, il y a dix ans et plus.

Iam annum hîc egi tertium, Iay desia demoure trois ans cyens.

Pridem, il y a fort long temps.

Non ita pridem, Il ny a pas fort long temps ${ }^{15}$.

Dans certains cas, le latin fautif est tellement français qu'il n'est pas traduit, mais directement corrigé, comme dans ce court paragraphe :

Quando loquebamur Gallicum, regens uenit quod non cogitabamus,

amendé en :

Cum Gallice fabularemur, praeceptor interuenit de improuiso.

bien plus correct pour le lexique et l'emploi du cum historicum au subjonctif ${ }^{16}$.

Le temps passant, Cordier a donc mis au point une série d'exercices progressifs qu'il réunit à la fin de sa vie en un seul ouvrage, les Colloquia Scholastica imprimés en 1564 chez Henri Estienne. La préface ne laisse pas de doute sur la nature de ce

attendre, tant que uous soyez reuenu; il complète ce dernier exemple de nouveau par de la synonymie : Varietas : expectare, praestolari, opperiri.

15 Mathurin Cordier, De... emendatione, Paris, Robert Estienne 1536, in- $4^{\circ}$, chapitre 2, $\$ 31$, p. 13.

16 Mathurin Cordier, De... emendatione, Paris, Robert Estienne 1536, in- $4^{\circ}$, chapitre 2, $\$ 166$, p. 28. 
livre : âgé de quatre-vingt-quatre ans, Cordier, qui mourra quelques mois plus tard, le présente comme un testament pédagogique où il a rassemblé la plupart des scènes de la vie quotidienne scolaire qui peuvent se tenir en latin. Ouvrage d'éducation morale également, les Colloquia sont organisés en quatre livres progressifs, où le premier met en scène les plus jeunes des élèves discutant entre eux de sujets ayant trait à la vie de la classe et du collège ; le livre 2 prolonge les mêmes thèmes avec des élèves plus âgés et des dialogues plus complexes. Le livre 3 met en scène le maître parlant avec les élèves, et le livre 4 est composé de dialogues longs, entre adolescents en fin de scolarité, qui proposent aussi des exemples moraux aux plus jeunes. Ces dialogues sont conçus comme des textes à apprendre, à jouer en classe et à utiliser en situation. Ils racontent le quotidien de leurs acteurs, se lever ou pas, être en retard, échanger livres et cahiers, manger, boire et même boire en cachette du surveillant, satisfaire ses besoins naturels, raconter ce qui se passe à la maison... Ce quotidien est rendu dans une langue fluide, éloignée du registre de la phrase complexe rhétorique, mais élégante et syntaxiquement impeccable. Cordier réalise parfaitement un idéal de latine loqui, qui, pour peu qu'il soit prononcé avec soin, pourrait donner une idée de l'oral quotidien de l'Antiquité classique.

Il me semble cependant qu'il faut apporter quelques nuances à la constatation de cette réussite. En effet, malgré les apparences, cette langue n'est pas réellement une langue de communication : elle est un exercice qu'il faut entretenir pour progresser, et même lorsqu'on n'a rien à se dire, il est bon de continuer la conversation pour prolonger le temps de pratique, et en fait, de parler pour parler... Cette idée est d'ailleurs explicitée par Cordier lui-même, tant dans les colloques mettant en scène les élèves les plus jeunes, à qui il faut donner de bonnes habitudes, que dans les textes visant les plus âgés, qu'il faut pousser à se perfectionner.

Par exemple le colloque 45 du livre 1 met en scène deux petits garçons, Galatinus et Burcardus, qui sont libérés de cours pour la récréation. Galatinus, qui est visiblement un élève modèle, propose à son camarade de "bavarder un peu ", paulisper confabulari, avant de se mettre à jouer. Convaincus qu'il vaut mieux commencer par ce «bavardage ", confabulatio, que par un jeu qu'ils auront du mal à interrompre, ce qui fait donc implicitement de la confabulatio un moment qui n'a rien de ludique, les deux enfants se font alors réciter mutuellement du vocabulaire latin ayant trait aux objets de la vie quotidienne et à la nourriture. Le dialogue se finit par une controverse sur la traduction de "sauce ", dont ils remettent l'arbitrage à plus tard, pour " ne pas perdre une occasion de jouer ${ }^{17}$ ". Ce texte montre clairement la confabulatio latine comme un exercice scolaire, qui n'est pas nécessairement porteur de communication : ce que ces enfants ont à se dire passera sans doute beaucoup plus dans l'heure de jeu que dans la récitation d'une liste de vocabulaire.

17 Mathurin Cordier, Colloquiorum scholasticorum libri quatuor, Genève, Henri Estienne, 1564, colloque 45 livre 1, p. 29-30. La controverse à propos de "sauce " est de savoir si la traduction en est condimentum ou intinctus. 
De même, le colloque 33 du livre 4 met en scène Athanase et Benjamin, nettement plus âgés que Galatin et Burcard, qui discutent de l'argent qu'un père peut donner à son fils et de la correction morale d'en demander ou pas, l'un poussant l'autre à ne pas laisser passer les occasions, alors que son camarade s'offusque de formuler une telle demande. Vers la fin du dialogue, nous comprenons cependant que cette légère controverse est feinte : Athanase est en fait d'accord avec Benjamin sur le fait qu'on ne demande pas d'argent à ses parents, et il a simplement alimenté la confabulatio en jouant un rôle; d'ailleurs il demande à Benjamin " ce qu'il pense de ce bavardage ». Benjamin ne peut que lui répondre « qu'il [leur] a donné un sujet assez adapté à [leur] moment de loisir du soir ", auquel le surveillant, même embusqué, n’aura rien à reprocher. Les lignes suivantes sont tout à fait édifiantes :

Athanase : Sans aucun doute, ce que notre maître nous répète souvent est bien vrai.

Benjamin : Quoi donc?

Athanase: Que l'abondance de la langue latine et la capacité de la parler s'acquièrent principalement par ces exercices : écrire souvent, bavarder ensemble, lire les auteurs, traduire du français en latin, ou du latin en français.

Benjamin : Exerçons-nous y donc avec diligence, avec l'aide de Dieu notre Seigneur, dans la main de qui sont déposées toutes nos études ${ }^{18}$.

Ces textes nous confirment bien que l'oral latin entre les enfants est un élément scolaire, et au mieux un jeu théâtral où on peut fabriquer un personnage de fiction, pourvu qu'il parle... Certains colloques, comme le 10 du livre 4, laissent même passer entre les lignes l'idée que si certains, qui savent du latin, cherchent des cachettes pour se raconter des « inepties » en français, c'est peut-être qu'il s'agit là de sujets de conversation pour lesquels le latin corseté du collège est inopérant ${ }^{19}$.

Cette langue, pratiquée dans le collège où pour les plus âgés on interdit le français, devient donc une langue intra muros, "entre les murs ", où le lieu scénique des dialogues est toujours l'intérieur de l'établissement scolaire. Les plus zélés peuvent commencer à s'exercer à la confabulatio sur le chemin qui les mène à l'école, mais ils courent le risque

18 Mathurin Cordier, Colloquiorum..., colloque 33 livre 4, p. 180 : Athanasius : sed quid censes de hac confabulatione? Beniaminus: Argumentum satis aptum dedisti nobis in hoc otio nostro vespertino. A. : Ecquid habuit sermo noster quod reprehendisset obseruator, si forte (ut solet) nos obseruasset ex insidiis? B. : Nibil, ut opinor. A. : Profecto verum est quod saepe nobis praeceptor inculcat. B. : Quid illud est? A. : Latinae linguae copiam et facultatem comparari his potissimum rebus, saepe scribendo, confabulando, legendis auctoribus, Gallica Latine, aut Latina Gallice conuertendo. B. : Ergo his rebus diligenter nos exerceamus, adiutore Domino Deo, in cuius manus sita sunt studia nostra omnia.

19 Mathurin Cordier, Colloquiorum..., colloque 10 livre 4, p. 158 : Edictum suum ad eos demum pertinere qui quum Latine sciant, tamen semper latibula quaerunt ut Gallice fabulentur, idque de rebus ineptissimis. ("Son édit [du précepteur] vise seulement ceux qui, alors qu'ils savent le latin, cherchent cependant constamment des cachettes pour parler français, et sur des sujets particulièrement ineptes. ") 
d'être la risée des chenapans qui ne font rien de bon, et, dit Michael dans le colloque $30 \mathrm{du}$ livre 2, "se moquent de nous à pleine bouche parce que nous parlons latin dans la rue ${ }^{20}$ ". L'exercice est donc incongru hors de l'espace scolaire, et si ce monde extérieur apparaît dans les dialogues, c'est en quelque sorte par échos : la maison, les rapports avec les parents, les activités commerciales ou agricoles de la famille ne sont pas absents, mais les enfants en parlent entre eux a posteriori, puisqu'ils en parlent au collège, pour se raconter la journée de la veille ou comparer le goûter qu'ils ont amené. Les confabulationes sont bien plus souvent occupées de questions purement scolaires, dont certaines reviennent plusieurs fois sous toutes les formes possibles et dans tout le recueil : la qualité du papier, des plumes, de l'encre et leur prix, comment demander une permission de sortie, comment et pourquoi le maître peut s'absenter un moment de sa classe sont des situations que les Colloquia représentent constamment avec les enfants les plus jeunes comme les plus âgés.

Cet univers confiné crée en conséquence un idiolecte technique que les personnages manient, au sens propre, comme un jargon, et qui comporte tout un lexique qui n'a jamais exactement été employé de cette façon dans un texte antique. Le lecteur des dialogues devient rapidement familier, tant ils apparaissent souvent, avec le praeceptor, maître principal et directeur de l'école, et son hypodidascalus, sorte de surveillant général qui s'occupe des pensionnaires, vérifie la présence ou l'absence des répétiteurs, délivre les bons de sortie... Ces deux termes sont cicéroniens et relèvent déjà de l'univers didactique dans les textes de l'Antiquité, mais il n'en va pas de même pour l'obseruator qui surveille les enfants et leurs conversations en latin, ou le nomenclator qui relève les absents, au moment où, le matin, catalogus recitatur, on fait l'appel ${ }^{21}$. Syntaxiquement impeccable, stylistiquement correcte, la langue des colloquia s'adapte à l'univers quotidien des enfants dans l'école du XVI siècle : elle tente de ressusciter un oral dont cependant nous n'avons aucune trace précise dans les textes de l'Antiquité, et pour lequel une réelle modélisation, telle qu'elle se pratique pour l'écrit, est impossible. La langue des Colloquia est alors autant une restauration qu'une invention, celle d'un idiolecte scolaire dont la technicité même confirme le statut d'exercice d'apprentissage, et non de langue de communication.

20 Mathurin Cordier, Colloquiorum..., colloque 30 livre 2, p. 66 : Etiam nos irrident plenis buccis quod Latine per uicos loquamur.

21 Hypodidascalus se trouve dans les Familiares, livre 9, lettre 18 ; le mot renvoie à l'univers précisément scolaire. Praeceptor est du champ sémantique de l'enseignement mais non spécifiquement de l'école ; les autres termes sont employés par glissement sémantique et accomodatio lexicale plutôt réussis. 


\section{Conclusion}

Mathurin Cordier n'a pas vu la postérité de son livre : il n'a pu en mesurer le succès fulgurant et généralisé, mais n'en a pas vu non plus une certain détournement. En effet une des clés de ce succès et de la multiplication des éditions en France, en Suisse, en Allemagne et en Angleterre notamment, jusqu'au XVIII siècle, a été la traduction en vernaculaire qui très rapidement accompagne le texte latin. Si la pérennité de ces éditions atteste l'immobilité de l'univers scolaire puisque les sujets des colloques sont toujours valides pour l'essentiel, l'introduction d'une traduction modifie sensiblement le propos. En effet, on trouve très vite, quelques années après les premières impressions, des éditions remaniées ou enrichies de dialogues qui ne sont pas de Cordier, mais où le texte latin est doublé d'un texte vernaculaire en vis-à-vis, ce qui change la nature de l'outil pédagogique ${ }^{22}$. En effet, Cordier avait chassé le français du livre comme du collège pour créer un outil d'apprentissage par immersion ; mais cet outil devient dans ces versions avec traduction un outil d'apprentissage par transfert, où le français revient comme support premier de compréhension. Ce résultat est la matérialisation tacite d'un échec pédagogique : il est impossible, à partir de la fin du XVI e siècle, d'apprendre le latin sans le français, même en recréant un monde protégé du vernaculaire entre les murs du collège. L'optimisme d'Érasme qui affirmait dans le De recta pronuntiatione que Quod olim fuit, instaurari potest, "ce qui a existé un jour peut se restituer ${ }^{23}$ " est donc quelque peu battu en brèche en ce qui concerne l'oral latin : le temps du latin vivant, langue de communication, est bien révolu, et le dévouement, voire le militantisme d'attachants pédagogues comme Mathurin Cordier n'est pas parvenu à endiguer le triomphe des vernaculaires. Ceux-ci, langues véritablement vivantes, ont lentement mais sûrement poussé le latin sur la voie de ce qu'il est aujourd'hui, c'est-à-dire un passionnant objet d'études, et une langue définitivement morte.

\begin{tabular}{l} 
Martine FURNO \\
\hline CERPHI, UMR 5037, Ens Lyon. \\
15 parvis René Descartes \\
F-69007 Lyon \\
martine.furno@ens-lyon.fr
\end{tabular}

22 Voir par exemple, pour le français, l'édition de François Forest pour la veuve de Jean Durant, Genève, 1593 (consultable en ligne sur e-rara). Ce travail soigné présente les colloques de l'édition de 1564, accompagnés de quelques autres probablement ajoutés par l'imprimeur, et où le français, sur la colonne extérieure de la page, double intégralement le latin relégué en colonne intérieure. D'autres éditions du même type existent pour l'allemand, à Bâle notamment, puis pour l'anglais.

23 Érasme, De recta... pronunciatione, p. 75. 
Une famille exemplaire : Mathurin Cordier, Colloquiorum scholasticorum libri quatuor, livre 2, colloque 44, Genève, Henri Estienne, 1564, p. 75-76.

Traduction M. Furno.

Colloquii XLIIII argumentum

Montanus et Eusebius conferunt annos suos.

Laudatur docti paedagogi diligentia, item boni patris prudentia in liberorum instituendorum cura. Agnoscitur in ea re Dei beneficium. EXEMPLVM exercitationis in lingua Latina a teneris annis.

Montanus, Eusebius.

Montanus: Quot annos habes?

Eusebius : Tredecim, ut a matre accepi, tu uero?

M. : Equidem non tot habeo.

E. : Quot igitur?

M. : Deest unus.

E. : Sunt ergo duodecim.

M. : In promptu est ratio.

E. : Sed frater tuus quotum agit annum?

M. : Quintum.

E. : Quid ais? Iam latine loquitur.

M. : Quid miraris ? Semper habemus domi paedagogum et doctum et diligentem : semper nos Latine loqui docet. Nibil Gallicum effert, nisi aliquid declarandi causa; quinetiam patrem non audemus nisi Latine alloqui.

E. : Nunquam igitur Gallice loquimini?
Argument du colloque 44

Montanus et Eusèbe comparent leur âge. On loue la diligence d'un pédagogue savant, et aussi la prudence d'un bon père dans le soin qu'il prend de l'éducation de ses enfants. On y reconnaît un bienfait de Dieu. Exemple d'exercice en latin dès les jeunes années.

Montanus : Quel âge as-tu ?

Eusèbe : treize ans, comme je l'ai appris de ma mère, et toi ?

M. : Je ne suis pas aussi âgé.

E. : Quel âge donc?

M. Un an de moins.

E. : Donc, douze ans.

M. : Le compte est clair.

E. : Mais ton frère, quel âge a-t-il ?

M. : Il va vers ses cinq ans.

E. : Quoi ? Il parle déjà latin!

M. : Pourquoi t'étonnes-tu ? Nous avons toujours à la maison un pédagogue docte et diligent : il nous enseigne toujours à parler latin, et ne nous dit rien en français, si ce n'est pour nous expliquer quelque chose ; bien plus, nous n'osons pas parler à notre père autrement qu'en latin.

E. : Vous ne parlez donc jamais français? 
M. : Solum cum matre, idque certa quadam hora, quum illa nos ad se vocari iubet.

E. : Quid agitis cum familia?

M. : Cum familia rarus est nobis sermo, et quidem tantum in transitu : et tamen famuli ipsi nos Latine alloquuntur.

E. : Quid ancillae?

M. : Si quando usus postulat ut eas alloquamur, vtimur sermone uernaculo, vt solemus cum ipsa matre.

E. : O vos felices, qui tam diligenter docemini!

M. : Est Deo gratia, cuius dono patrem habemus, qui curet nos tam accurate erudiendos.

E. : Certe eius rei laus et honor vnico caelesti Patri debetur.

M. : Sed quid agimus? Iam audio recitari catalogos.

E. : Ergo festinemus.
M. : Seulement avec notre mère, à des moments précis, quand elle ordonne que nous soyons appelés auprès d'elle.

E. : Que faites-vous avec les domestiques?

M. : Avec les domestiques, nous avons rarement de conversation, et seulement au passage ; et cependant les serviteurs mêmes nous parlent latin.

E. : Et les servantes ?

M. : Quand il est utile que nous leur parlions, nous utilisons le vernaculaire, comme nous en avons l'habitude avec notre mère.

E. : Que vous êtes heureux, d'être éduqués avec tant de diligence!

M. : C'est grâce à Dieu, qui nous a donné un père qui se soucie avec autant de soin de nous rendre savants.

E. : Certes, la louange et l'honneur en sont dus au seul Père céleste.

M. : Mais que faisons-nous ? J'entends déjà qu'on fait l'appel.

E. : Hâtons-nous ! 